\title{
Evaluation of mandibular condyles in children with unilateral posterior crossbite
}

\section{Edson ILLIPRONTI-FILHO(a) Solange Mongelli de FANTINI(b) Israel CHILVARQUER ${ }^{(a)}$}

(a) Universidade de São Paulo - USP, School of Dentistry, Department of Stomatology, São Paulo, SP, Brazil.

(b) Universidade de São Paulo - USP, School of Dentistry, Department of Orthodontics and Dental Pediatrics, São Paulo, SP, Brazil.

Declaration of Interests: The authors certify that they have no commercial or associative interest that represents a conflict of interest in connection with the manuscript.

\section{Corresponding Author:}

Edson Illipronti-Filho

E-mail: illipronti@usp.br

DOI: 10.1590/1807-3107BOR-2015.vol29.0049

Submitted: May 20, 2014

Accepted for publication: Dec 10, 2014

Last revision: Feb 09, 2015

\begin{abstract}
The relationship of mandibular condyle dimensions and its association with unilateral posterior crossbite (UPXB) has been suggested in the literature. The purpose of this prospective study was to evaluate mandibular condyles on the left and right sides and between crossed and non-crossed sides in the sagittal and coronal planes, using cone-beam computed tomography (CBCT). Twenty CBCT images of 40 temporo mandibular joints (TMJs) in individuals in mixed dentition phase, which included 9 males (mean 7.9 years) and 11 females (mean 8.2 years), with unilateral posterior crossbite without premature contacts and functional mandibular shifts and with transverse maxillary deficiency. The criteria for sample exclusion included the presence of painful symptoms, facial trauma history, systemic diseases such as juvenile rheumatoid arthritis, mouth opening limitation ( $<40 \mathrm{~mm}$ ), congenital or genetic anomalies, and skeletal asymmetries that may result in TMJ disorders. Dimensional measurements of the condyles between the right and left sides and crossed and non-crossed sides in sagittal and coronal view were made. There was no significant difference between the measurements of the crossed and non-crossed sides in both sagittal and coronal view. These findings suggest that the presence of unilateral posterior crossbite in children with UPXB did not result in changes between the mandibular condyles in the right and left sides or between the crossed and non-crossed sides in the coronal or sagittal plane.
\end{abstract}

Keywords: Malocclusion; Mandibular Condyle; Cone-Beam Computed Tomography.

\section{Introduction}

The temporo mandibular joint (TMJ) region, more specifically the condyles, may undergo changes and remodeling according to the malocclusion present. This important joint involves not only mandibular movements during chewing, deglutition, and phonation but also the region where mandibular growth is expressed. The presence of any change, whether dimensional or positional, may affect not only its morphology but also compromise the facial cranial development taking place during that period. ${ }^{1,2}$

Unilateral posterior crossbite (UPXB) are malocclusions frequently found during the mixed dentition phase (11\%-20\%). ${ }^{3,4}$ It is a transversal 
problem that may involve one or more elements and is characterized by the inversion of the lingual vestibule relation of the upper and lower posterior teeth. UPXBs are classified into skeletal, dental, or functional, and may be uni- or bilateral. Perpetuation of that malocclusion in adult ages or even in young adults may cause changes both to the face, such as those seen in the TMJ. Considering that the possibility of spontaneous correction of that type of malocclusion is practically unfeasible, the diagnosis and treatment must occur as soon as possible. . $, 6,7^{-7}$

Changes in morphology of mandibular condyles, articular fossa, and its associations with the existing malocclusion are broadly reported in the phase of permanent dentition; however, few studies in the literature report the relation of mandibular condyle dimensions and its association with UPXB, and a vast majority of studies assess the mandibular condyle position relative to the mandibular fossa. ${ }^{8,9,10}$ Imaging exams have been used in the identification of structural and positional changes, which is similar to the bone contour of mandibular condyle and articular fossa, in the articular inter-relations and in the assessment of articular spaces considering the verification of possible changes in the beginning and end of the orthodontic treatment. ${ }^{11}$

Images obtained by cone-beam computed tomography $(\mathrm{CBCT})$ were shown to be a great method in the assessment of bone structures comprising the TMJ, mainly of the mandibular condyle and articular fossa. This imaging technique allows both linear and a volumetric measurement of the structures described and has high accuracy. ${ }^{12,13}$

The aim of this study was to evaluate if the presence of unilateral posterior crossbite in children during the mixed dentition period could lead to alterations in dimensional mandibular condyles. This was performed by CBCT.

\section{Methodology}

The sample consisted of 20 CBCT images of 40 TMJs of subjects in the mixed dentition phase, which included 9 males (mean age 7.9 years) and 11 females (mean age 8.2 years), with UPXB without premature contacts and functional mandibular shifts and with transverse maxillary deficiency.
Images were obtained for diagnosis, planning, and treatment of this malocclusion. Criteria adopted for sample exclusion included the presence of painful symptoms, facial trauma history, systemic diseases such as juvenile rheumatoid arthritis, mouth opening limitation $(<40 \mathrm{~mm})$, congenital or genetic anomalies, and skeletal asymmetries that may result in TMJ disorders. The data was collected in each participant's initial clinical assessment file.

The sample size was calculated to observe the difference between the measures in crossed and non-crossed sides. A power of $90 \%$ was assumed and $5 \%$ level of significance was set in both sagittal and coronal cuts. The difference in the measures was around $10 \%$. In sagittal cut, the mean was 6.6 in the non-crossed side and standard deviation (SD) was 0.3. The SD was 0.6 in the crossed side. Thus, an $n$ of 18 subjects was determined. In coronal cuts, the mean was 14 and SD was 1.3 in non-crossed sides SD was 1.4 and, the $n$ of 20 subjects. For simplification, the sample size was standardized to 20 participants.

The study was approved by the Research Ethics Committee of the Universidade de São Paulo - USP, School of Dentistry (numbers 200/06 and 16/2008).

The measurements obtained in this study were conducted prior to the UPXB correction orthodontics treatment, repeated three times, and performed by one single examiner, with a 1-week interval between the times proposed. The variance analysis test was applied to assess the reliability of intra-examiner results.

All images were obtained with an i-CAT-KaVo - 3D Dental Imaging System (Imaging Sciences International, Kavo Group, Hatfield, USA), with a field of view (FOV) of $13 \mathrm{~cm} \times 17 \mathrm{~cm}$, with automatic collimation, acquisition time between 5 and $26 \mathrm{~s}$, and pulse exposure in the 14-bit gray scale and 16,384 shades of gray. The focal point was $0.5 \mathrm{~m}$ with a 0.3 $\mathrm{mm}$ voxel. Effective dose of $36.74 \mathrm{uSv}$ and cylindrical reconstruction, being primary with scan obtainment from 20 seconds to 1 minute, and secondary in real time. Prior to the CBCT acquisition, the patient was positioned seated in the device. For better control in capturing images, the head was stabilized to obtain the Frankfurt plane parallel to the ground and then the tomographic sensor was positioned to capture the image of the entire head. The patient was instructed to 
remain still, with lips relaxed, and to avoid deglutition during image acquisition. After capturing attenuated X-rays, the data was digitalized by tomography sensors through reconstruction algorithms and converted to the Digital Imaging and Communication in Medicine (DICON) format. After capturing the images, primary image reconstruction was performed at the workstation. After confirmation of the accurate obtainment of the reconstructed image, the patient was released. Software Dolphin 3D ${ }^{\circledR}$ (Dolphin Imaging/Patterson Dental, Chatsworth, USA) was used to perform proposed measurements. Prior to measuring the mandibular condyle dimensions, images were standardized according to the cranial positioning orientation. ${ }^{13}$ In Figure 1, the axial plan coincides with the orbital points; in the side images, the coronal plan coincides with the Porion (Po), in the left and right sides, and the axial plan is superimposed to the Frankfurt plan; the median sagittal plan unites the Nasion $(\mathrm{N})$ and the anterior nasal spine (ANS).

In the Dolphin software, we accessed the Build X-ray, selected the TMJ imaging screen, performed orientation of the TMJ cut, and then captured images of the mandibular condyles in the respective articular fossa. To obtain measurements, we adopted the criteria developed by Schluter et al. ${ }^{14}$ as follows:

- M (medial face of the condyle): Anatomic point representing the most medial position of the mandibular condyle in coronal cut;
- L (lateral face of the condyle): Anatomic point representing the most lateral position of the mandibular condyle in coronal cut;

- A (anterior face of the condyle): Anatomic point representing the most anterior position of the mandibular condyle in sagittal cut;

- $\mathbf{P}$ (posterior face of the condyle): Anatomic point representing the most posterior position of the mandibular condyle in sagittal cut;

- M-L (mandibular condyle width): Distance between anatomic points $\mathbf{M}$ and $\mathbf{L}$ in coronal cut;

- A-P (Mandibular condyle length): Distance between anatomic points $\mathbf{A}$ and $\mathbf{P}$ in sagittal cut. Figures 2 and 3.

All values obtained were tabulated for analysis with the appropriate statistical tests, and average values and standard deviations were obtained separately for crossed and non-crossed sides as well as for the right and left sides, both in coronal and sagittal cuts.

\section{Results}

We used variance analysis (ANOVA) for repeated measurements for error assessment of the intra-examiner method. The descriptive analysis of data and result of the model applied are presented in Table 1, showing that at the 5\% significance level, there is no method error for the single examiner in the three measurements in any of the positional

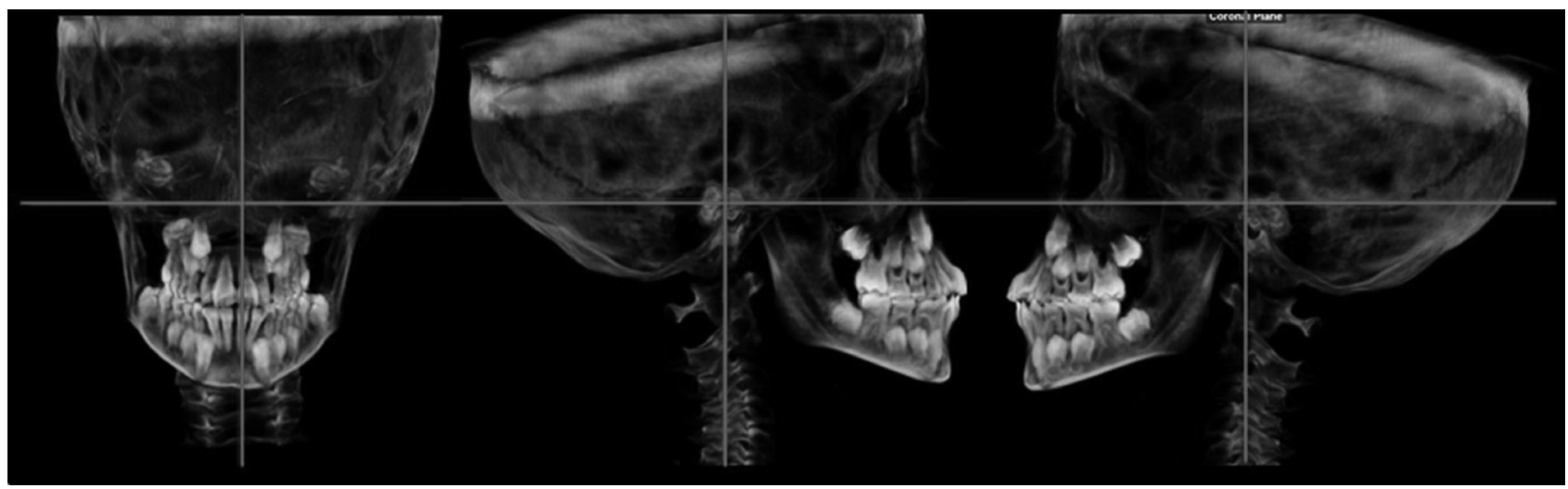

Figure 1. Cranial positioning orientation using Software Dolphin 3D ${ }^{\circledR}$ (Dolphin Imaging/Patterson Dental, Chatsworth, USA). 


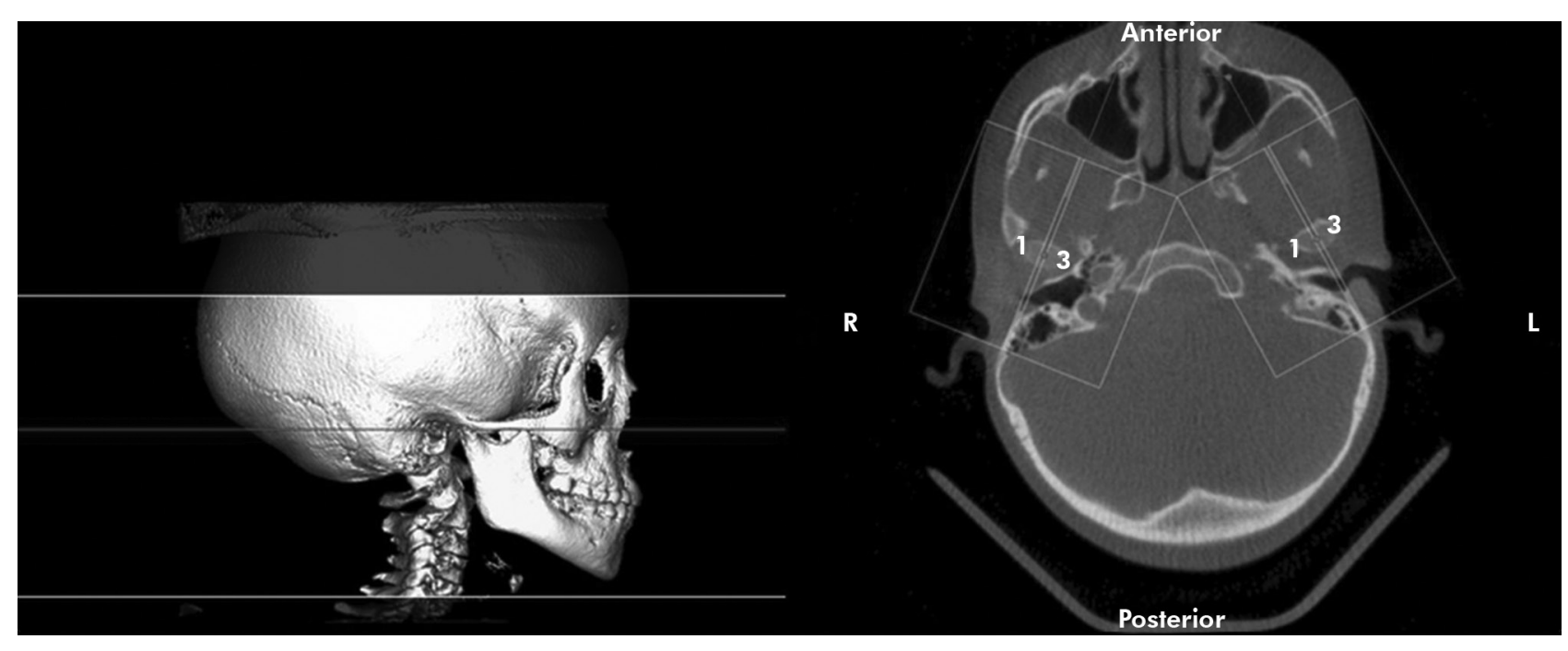

Figure 2. Cuts Orientation - Software Dolphin 3D ${ }^{\circledR}$ (Dolphin Imaging/Patterson Dental, Chatsworth, USA).

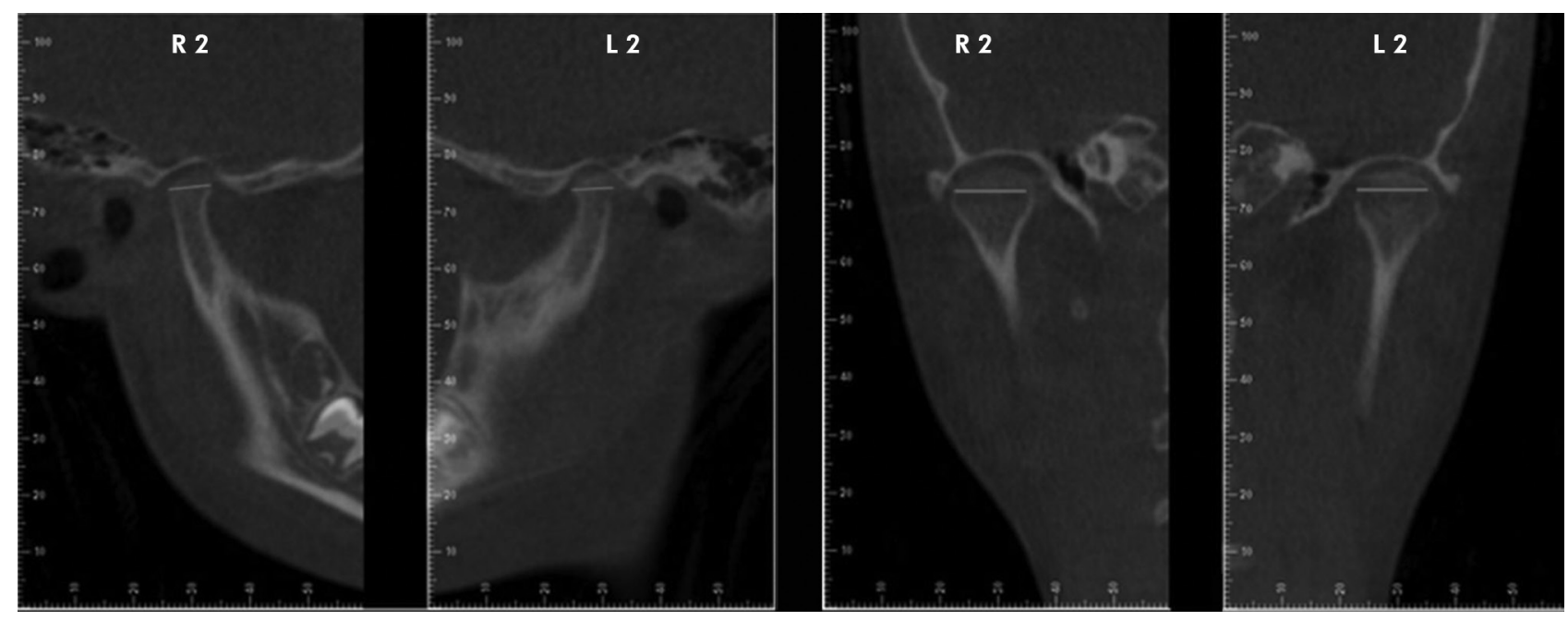

Figure 3. Measures in Sagittal and Coronal Planes - Software Dolphin 3D ${ }^{\circledR}$ (Dolphin Imaging/Patterson Dental, Chatsworth, USA).

images that were captured: crossed sagittal side, non-crossed sagittal side, crossed coronal side, non-crossed coronal side.

The descriptive analysis of the dimensional measurements of the mandibular condyle between the crossed and non-crossed sides in sagittal and coronal cuts, and the results obtained by the Paired t-Student parametric test are shown in Table 2. It was noted that at the 5\% significance level, there is no significant difference in the dimensional measurements between the crossed and non-crossed sides in sagittal and coronal cuts.

\section{Discussion}

UPXB are described as predisposing factors for changes in the mandibular condyle morphology and may affect cranial facial development. During the mixed dentition period, cranial facial development is expressed singularly and $30 \%-40 \%$ of all development occurs during this phase. Thus, in addition to establish favorable occlusal relations, the primary condition to obtain images involves the balance of stomatognathic functions such as chewing, phonation, deglutition, and breathing. ${ }^{4,7,9}$ 
Table 1. Descriptive analysis of data and results of three measurements to assess intraexaminer errors using model analysis of variance (ANOVA) for repeated measures.

\begin{tabular}{|c|c|c|c|c|}
\hline Variable & Measure 1 & Measure 2 & Measure 3 & $\mathrm{p}$-value \\
\hline \multicolumn{5}{|l|}{ Crossed side - Sagital } \\
\hline Mean \pm Standard deviation & $6.63 \pm 0.89$ & $6.59 \pm 0.89$ & $6.53 \pm 0.81$ & 0.254 \\
\hline Median (Minimum - Máximum) & $6.55(4.5-8.6)$ & $6.55(4.4-8.4)$ & $6.55(4.5-8.3)$ & \\
\hline Total & 20 & 20 & 20 & \\
\hline \multicolumn{5}{|l|}{ Non-crossed side - Sagital } \\
\hline Mean \pm Standard deviation & $6.71 \pm 0.64$ & $6.61 \pm 0.72$ & $6.58 \pm 0.71$ & 0.230 \\
\hline Median (Minimum - Maximum) & $6.8(5.4-8.1)$ & $6.7(5.4-7.9)$ & $6.65(5.3-8)$ & \\
\hline Total & 20 & 20 & 20 & \\
\hline \multicolumn{5}{|l|}{ Crossed side - Coronal } \\
\hline Mean \pm Standard deviation & $14.22 \pm 1.97$ & $14.09 \pm 1.64$ & $14 \pm 1.8$ & 0.261 \\
\hline Median (Minimum - Maximum) & $14.35(11.1-18.8)$ & $14(11.9-18.4)$ & $13.9(10.9-18.6)$ & \\
\hline Total & 20 & 20 & 20 & \\
\hline \multicolumn{5}{|l|}{ Non-crossed Side - Coronal } \\
\hline Mean \pm Standard deviation & $14.8 \pm 1.9$ & $14.46 \pm 1.75$ & $14.42 \pm 1.81$ & 0.051 \\
\hline Median (Minimum - Maximum) & $14.65(12-18.8)$ & $14.35(12.1-18.4)$ & $14.1(11.8-18.3)$ & \\
\hline Total & 20 & 20 & 20 & \\
\hline Total & 20 & 20 & 20 & - \\
\hline
\end{tabular}

Table 2. Descriptive analysis, mandibular condyle between the crossed and non-crossed side, in the sagittal and coronal planes, and the result of parametric test t-Student test.

\begin{tabular}{|c|c|c|c|}
\hline Variable - Side & Crossed & Non-crossed & $p$-value \\
\hline \multicolumn{4}{|l|}{ Sagital } \\
\hline Mean \pm Standard deviation & $6.58 \pm 0.85$ & $6.63 \pm 0.66$ & 0.645 \\
\hline Median (Minimum - Maximum) & $6.57(4.47-8.43)$ & $6.75(5.4-8)$ & \\
\hline Total & 20 & 20 & \\
\hline \multicolumn{4}{|l|}{ Coronal } \\
\hline Mean \pm Standard deviation & $14.1 \pm 1.78$ & $14.56 \pm 1.79$ & 0.051 \\
\hline Median (Miminum - Maximum) & $14.18(11.3-18.6)$ & $14.35(12.1-18.5)$ & \\
\hline Total & 20 & 20 & - \\
\hline
\end{tabular}

In contrast to our findings, a study on the morphological features of dentition and palate in subjects with skeletal asymmetries reported that there is a significant correlation between the lateral mandibular deviation, present to some degree in the UPXB, and the morphology, both in the palate and other structures such as the mandibular condyle. ${ }^{15}$ However, it should be noted that only patients with non-functional UPXB were studied. Kiliç et al. ${ }^{16}$ showed that patients with UPXB have asymmetric mandibular condyles on panoramic radiographs, which may contribute to a skeletal mandibular asymmetry. During the growth period, mandibular condyle displacement in the articular fossa induces differential growth. Hence, it is likely that UPXBs may induce asymmetries both in the mandibular condyle and on the face. Our results show that UPXB results in numerically different values between the crossed and non-crossed sides; however, it was not possible to confirm that such difference in values could lead to an actual asymmetry of mandibular condyle.

In a study conducted using CBCT in the assessment of volume and stresses on the mandibular condyle in children with juvenile idiopathic arthritis, Huntjens et al. ${ }^{17}$ concluded that CBCT is an accurate method to make such measurements (volumetric 
and linear) on the mandibular condyles and that the asymmetry degree was variable and frequent in the majority of the sample. Similarly, our results show that the CBCTs presented good accuracy and excellent quality.

Studies by Pinto et al..$^{18}$ and Kiki et al. ${ }^{19}$ demonstrated a relationship between the crossed and non-crossed sides as well as the presence of condylar asymmetry, both in numeric values when comparing crossed and non-crossed sides; however, no statistical significance was noted. These results agree with our findings despite different methodologies used, i.e., submento vertex projection radiography and panoramic radiography.

It is suggested in the literature that the posterior crossbite, whether functional or skeletal should be corrected even in the primary dentition. However, when corrected during the mixed dentition period (age 7-9 years), the results on follow-up proved to be more stable, whereas phase recurrences are more frequent in advancing years during primary dentition. ${ }^{20,21}$ The cost-benefit relationship should be taken into consideration because corrective treatment can help in eliminating the functional deviations and possible asymmetries in both the condyle, the mandibular ramus, and of the face. ${ }^{22}$

\section{References}

1. Hegde S, Praveen BN, Shetty SR. Morphological and radiological variations of mandibular condyles in health and diseases: a systematic review. Dentistry. Dec 2013;3(1):01-05. doi:10.4172/2161-1122.1000154.

2. Mongini F, Schmid W. Treatment of mandibular asymmetries during growth. A longitudinal study. Eur J Orthod. 1987 Feb;9(1):51-67.

3. Schröder U, Schröder I. Early treatment of unilateral posterior crossbite in children with bilaterally contracted maxillae. Eur J Orthod. 1984 Feb;6(1):65-9.

4. Kutin G, Hawer RR. Posterior cross-bite in the deciduous and mixed dentition. Am J Orthod. 1969 Nov;56(5):491-504.

5. Myers DR, Barenie JT, Bell RA, Williamson EH. Condylar position in children with functional posterior crossbites: before and after crossbite correction. Pediatr Dent. 1980;9:51-67.

6. Lam PH, Sadowsky C, Omerza F. Mandibular asymmetry and condylar position in children with unilateral posterior crossbite. Am J Orthod Dentofacial Orthop. 1999 May;115(5):559-75.
Despite the assessment of volume and stresses on the mandibular condyle in young adults, who did not show pain or dysfunction of TMJ, Tecco et al. ${ }^{23}$ reported no statistically significant differences between the crossed and non-crossed sides. They also indicate that there is a statistical difference between genders, males presenting a slightly higher volume compared to females.

Even the results of our study, using a sample with young children (mean age 8.6 years), show that there was no statistically significant difference between crossed and non-crossed sides. Based on the literature describing asymmetries between the left and right sides and crossed and non-crossed sides in patients with UPXB, we understand that if the malocclusion is not corrected accordingly, these asymmetries may become evident with natural development.

\section{Conclusion}

The results of the present study show that the presence of unilateral posterior crossbite in children did not result in changes between the right and left mandibular condyles, between right and left sides, or between crossed and non-crossed sides in sagittal or coronal plans.

7. Rodrigues AF, Fraga MR, Vitral RWF. Computed tomography evaluation of the temporomandibular joint in Class I malocclusion patient: condylar symmetry and condyle-fossa relationship. Am J Orthod Dentofacial Orthop. 2009 Aug;136(2):192-8.

8. Katsavrias EG, Halazonetis DJ. Condyle and fossa shape in Class II and Class III skeletal patterns: a morphometric tomographic study. Am J Orthod Dentofacial Orthop. 2005 Sep;128(3):337-46.

9. Pirttiniemi P, Kantomaa T, Lahtela P. Relationship between craniofacial and condyle path asymmetry in unilateral crossbite patients. Eur J Orthod. 1990 Nov;12(4):408-13.

10. Kurusu A, Horiuchi M, Soma K. Relationship between occlusal force and mandibular condyle morphology. Evaluated by limited cone-beam computed tomography. Angle Orthod. 2009 Nov;79(6):1063-9.

11. Kluemper TG, Beeman CS, Hick Ep. Early orthodontic treatment: what are the imperative? J Am Dent Assoc. 2000 May;131(5):613-20. 
12. Seth V, Kamath P, Vaidya N. Cone beam computed tomography: third eye in diagnosis and treatment planning. Int J Orthod Milwaukee. 2012 Summer;23(2):17-22

13. Ribeiro AN, Paiva JB, Rino-Neto J, Illipronti-Filho E, Trivino T, Fantini SM. Upper airway expansion after rapid maxillary expansion evaluated with cone beam computed tomography. Angle Orthod. 2012 May;82(3):458-63. DOI: 10.2319/030411-157.1. Epub 2011 Oct 17.

14. Schlueter B, Kim KB, Oliver D, Sortiropoulos G. Cone beam computed tomography $3 \mathrm{D}$ reconstruction of the mandibular condyle. Angle Orthod. 2008 Sep;78(5):880-8.

15. Hayashi K, Muguruma T, Hamaya M, Mizoguchi I. Morphologic characteristics of the dentition and palate in cases of skeletal asymmetry. Angle Orthod. 2004 Feb;74(1):26-30.

16. Kiliç N, Kiki A, Oktay H. Condylar asymmetry in unilateral posterior crossbite patients. Am J Orthod Dentofacial Orthop. 2008 Mar;133(3):382-7.

17. Huntjens E, Kiss G, Wouters C, Carels C. Condylar asymmetry in children with juvenile idiopathic arthritis assessed by cone-beam computed tomography. Eur J Orthod. 2008 Dec;30(6):545-51.
18. Pinto AS, Buschang PH, Throckmorton GS, Chen P. Morphological and positional asymmetries of young children with functional unilateral posterior crossbite. Am J Orthod Dentofacial Orthop. 2001 Nov;120(5):513-20.

19. Kiki A, Kiliç M, Oktay H. Condylar asymmetry in bilateral posterior crossbite patients. Angle Orthod. 2007;77(1):77-81.

20. O’Byrn BL, Sadowsky C, Schneider B, BeGole EA. An evaluation of mandibular asymmetry in adults with unilateral posterior crossbite. Am J Orthod Dentofacial Orthop. 1995 Apr;107(4):394-400.

21. Harrison JE, Ashby D. Orthodontic treatment for posterior crossbites. Cochrane Database Syst Rev. 2001;(1):CD000979.

22. Uysal T, Sisman Y, Kurt G, Ramoglu SI. Condylar and ramal vertical asymmetry in unilateral and bilateral posterior crossbite patients and a normal occlusion sample. Am J Orthod Dentofacial Orthop. 2009 Jul;136(1):37-43.

23. Tecco S, Saccucci M, Nucera R, Polimeni A, Pagnoni $M$, Cordasco G, et al. Condylar volume and surface in Caucasian young adult subjects. BMC Med Imaging. 2010 Dec;10:28. 